\title{
The $D$ coefficient in neutron beta decay in effective field theory
}

\author{
Shung-ichi Ando,, * Judith A. McGovern, ${ }^{1, \oplus}$ and Toru Sato 2 , \\ ${ }^{1}$ Theoretical Physics Group, School of Physics and Astronomy, \\ The University of Manchester, Manchester, M13 9PL, UK \\ ${ }^{2}$ Department of Physics, Osaka University, Toyonaka, \\ Osaka 560-0043, Japan
}

(Dated: October 30, 2018)

\begin{abstract}
In this paper we explore the time-reversal-odd triple-correlation coefficient in neutron beta decay, the so-called " $D$ coefficient", using heavy-baryon effective field theory with photon degrees of freedom. We find that this framework allows us to reproduce the known results for the contribution which comes from final-state interactions, and also to discuss higher-order corrections. In particular we are able to show that in the heavy-baryon limit all electromagnetic contributions vanish. By calculating the leading correction to the known result, we give a final expression which is accurate to better than $1 \%$. Hence we extend downwards the range over which the $D$ coefficient could be used to explore time-reversal violation from new physics.
\end{abstract}

PACS numbers: 12.40.Ks, 13.30.Ce, 23.40.-s

*Electronic address: shung-ichi.ando@manchester.ac.uk

${ }^{\dagger}$ Electronic address: judith.mcgovern@manchester.ac.uk

${ }^{\ddagger}$ Electronic address: tsato@phys.sci.osaka-u.ac.jp 


\section{INTRODUCTION}

In the expression for the polarised-neutron beta-decay rate, one of the terms which can be written is a triple scalar product of the neutron spin polarization and the momenta of the electron and neutrino; its coefficient is referred to as the " $D$ coefficient" [1]. Such a term is time-reversal-odd and, in the absence of final-state interactions, a non-zero coefficient would indicate the presence of time-reversal violating interactions in the beta-decay vertex.

An estimate of the $D$ coefficient from CP-violation in the standard model turns out to be extremely tiny, $D_{\mathrm{SM}} \leq 10^{-12}[2]$, whereas a prediction from, e.g., the minimal supersymmetric standard model (MSSM) is reported as $D_{\mathrm{MSSM}} \sim 10^{-7}[3]$ and more exotic mechanisms such as leptoquarks could give even larger results [4]. Experimental measurements of the $D$ coefficient may soon probe these values; recent papers give bounds of $D=[-0.6 \pm 1.2$ (stat) \pm 0.5 (syst) $] \times 10^{-3}$ from the EMIT experiment [5] and $D=[-2.8 \pm 6.4$ (stat) \pm 3.0 (syst) $] \times 10^{-4}$ from the Trine collaboration [6]; for a review of the future experimental prospects see, e.g., Ref. 7]. Increasingly precise measurements could open a new era for studies of physics beyond the standard model. A complication is that there are small but non-zero corrections from the electromagnetic final-state interaction [1]. The correction at the one-photon loop order vanishes in the zero-recoil approximation, and (relaxing that approximation) has been estimated by Callan and Treiman as $D_{\mathrm{FS}} \simeq 10^{-5}[8]{ }^{1}$ If new physics which contributes at or below this magnitude is to be explored, it would be desirable to know this value to an accuracy of $1 \%$ or less. As previously established in many other processes, the ideal tool for such work is low-energy effective field theory (EFT) [10].

In this work, we calculate the $D$ coefficient employing a pionless heavy-baryon EFT based on heavy-baryon chiral perturbation theory $(\mathrm{HB} \chi \mathrm{PT})$ with photon fields [11, 12, 13]. $\mathrm{HB} \chi \mathrm{PT}$ is a low-energy effective field theory of QCD and has a systematic expansion scheme (counting rules) in terms of small external momenta, symmetry-breaking terms (proportional to the pion mass, $m_{\pi}$ ), and the number of loops. "Small" is in relation to the scale $\Lambda_{\chi} \approx m_{\rho}$, which characterises the physics which is not explicitly included. "Heavy-baryon" indicates the approach in which the nucleon mass $M_{N}$ is also treated as a "large" scale, $M_{N} \approx$ $\Lambda_{\chi}$. Hence the usual expansion parameter of $\mathrm{HB} \chi \mathrm{PT}$ is roughly of order $m_{\pi} / M_{N}$. (For a recent review see Ref. [14].) However, because the typical energy of neutron beta decay is so small compared even to $m_{\pi}$, the pion itself can be integrated out and is not included as an explicit degree of freedom. The counting rules for neutron decay in the pionless EFT have been worked out by Ando et al [15]. We employ $\alpha / 2 \pi$ (which governs radiative corrections associated with photon loops), and $\bar{Q} / M_{N}$ (from the heavy-baryon expansion) as our expansion parameters, where $\alpha$ is the fine structure constant and $\bar{Q}$ is a typical momentum of neutron beta decay, $\bar{Q} \sim m_{n}-m_{p}-m_{e}\left(m_{n}, m_{p}\right.$ and $m_{e}$ are the neutron, proton, and electron mass, respectively). These two expansion parameters are numerically almost the same, $\alpha / 2 \pi \sim \bar{Q} / M_{N} \sim 10^{-3}$.

As we will show the Callan-Treiman result is reproduced in the EFT framework as the $\mathcal{O}\left(\alpha \bar{Q} / M_{N}\right)$ contribution, ${ }^{2}$ but the counting suggests that $\mathcal{O}\left(\alpha^{2} / 2 \pi\right)$ contributions could be as large. Such contributions coming from repeated Coulomb final-state interactions have

1 For a historical review, see Ref. [9]. This result has been confirmed by Refs [16, 17, 18]

${ }^{2}$ Since, as we will see, the $D$ coefficient requires a loop integral with an imaginary part, contributions to it will be generically a factor of $2 \pi$ larger than other terms at the same loop or $1 / M_{N}$ order, which we will keep track of: hence $\mathcal{O}\left(\alpha \bar{Q} / M_{N}\right)$ rather than $\mathcal{O}\left(\alpha \bar{Q} / 2 \pi M_{N}\right)$. 
already been shown to vanish for zero recoil [16], but radiative effects have not. However we are able to show that in the limit $M_{N} \rightarrow \infty$ all electromagnetic contributions to the $D$ coefficient vanish, and so corrections to the Callan-Treiman result must be higher order.

In fact the counting rules discussed above, while valid for the first three orders, must be modified at the order at which the counterterms encode information about the pions which have been integrated out. In fact the leading higher-order correction to the $D$ coefficient, which we calculate, comes from the pseudoscalar form factor of the weak nucleon current, which is enhanced because its scale is governed not by the nucleon mass but by the pion mass [19]. This allows us to conclude that the residual error is of the order of 1 part in $10^{3}$, and certainly smaller than $1 \%$.

\section{THE $D$ COEFFICIENT AND EFFECTIVE LAGRANGIAN}

The general expression for the differential neutron decay rate $d \Gamma$ is well known for the case wherein only the neutron is polarized:

$$
\begin{aligned}
\frac{d \Gamma}{d E_{e} d \Omega_{\hat{p}_{e}} d \Omega_{\hat{p}_{\nu}}} \simeq & \frac{\left(G_{F} V_{u d}\right)^{2}}{(2 \pi)^{5}}\left(1+3 g_{A}^{2}\right)\left|\vec{p}_{e}\right| E_{e} E_{\nu}^{2} \\
& \times\left(1+a \frac{\vec{p}_{e} \cdot \vec{p}_{\nu}}{E_{e} E_{\nu}}+\hat{n} \cdot\left(A \frac{\vec{p}_{e}}{E_{e}}+B \frac{\vec{p}_{\nu}}{E_{\nu}}+D \frac{\vec{p}_{e} \times \vec{p}_{\nu}}{E_{e} E_{\nu}}\right)\right),
\end{aligned}
$$

where $G_{F}$ is the Fermi constant, $V_{u d}$ is a CKM matrix element, and $g_{A}$ is the axial-current coupling constant. Here $E_{e}$ and $\vec{p}_{e}\left(E_{\nu}\right.$ and $\left.\vec{p}_{\nu}\right)$ are the electron (neutrino) energy and momentum, $\hat{n}$ is the neutron spin polarization vector, and $a, A, B, D$ are the correlation coefficients. The standard lowest order expressions for the correlation coefficients are

$$
a=\frac{1-g_{A}^{2}}{1+3 g_{A}^{2}}, \quad A=\frac{-2 g_{A}^{2}+2 g_{A}}{1+3 g_{A}^{2}}, \quad B=\frac{2 g_{A}^{2}+2 g_{A}}{1+3 g_{A}^{2}}, \quad D=0 .
$$

We can reproduce these expressions for the coefficients in the leading order (LO) EFT calculation. In addition, at NLO the $\mathcal{O}(\alpha / 2 \pi)$ radiative corrections and the $\mathcal{O}\left(\bar{Q} / M_{N}\right)$ recoil corrections (including the weak magnetism term) to the decay rate $\Gamma$ and correlation coefficients $a, A$, and $B$ have already been reported in Ref. [15]. A non-zero contribution to the $D$ coefficient, due to the electromagnetic final-state interaction, has been obtained by Callan and Treiman from one-photon-loop contributions [8] and we will see that the same expression comes out at NNLO.

The standard chiral counting rules for $\mathrm{HB} \chi \mathrm{PT}$ with and without photons are discussed in Refs. [11, 12, 13]. However as mentioned above, and discussed in more detail in Ref. [15], the smallness of the energy released in neutron beta decay modifies the counting so that we have two parameters, $\bar{Q} / M_{N} \simeq \bar{Q} / \Lambda_{\chi}$ and $\alpha / 2 \pi$, which are is numerically of the same magnitude. Thus we treat them as a single common expansion parameter in defining LO, NLO, etc.

The effective Lagrangian for the calculation of the $D$ coefficient in neutron beta decay reads [15]

$$
\mathcal{L}_{\beta}=\mathcal{L}_{e \nu \gamma}+\mathcal{L}_{N N \gamma}+\mathcal{L}_{N N e \nu},
$$

where $\mathcal{L}_{e \nu \gamma}$ is the standard Lagrangian for electron, neutrino, and photon, $\mathcal{L}_{N N \gamma}$ is the heavybaryon Lagrangian for nucleon interaction with a photon up to NLO (the $1 / M_{N}$ order), and 
$\mathcal{L}_{N N e \nu}$ is the $V-A$ interaction Lagrangian between the nucleon and lepton currents up to NLO:

$$
\begin{aligned}
\mathcal{L}_{e \nu \gamma}= & -\frac{1}{4} F^{\alpha \beta} F_{\alpha \beta}-\frac{1}{2 \xi_{A}}(\partial \cdot A)^{2}+\bar{\psi}_{e}\left(i \gamma \cdot D-m_{e}\right) \psi_{e}+\bar{\psi}_{\nu} i \gamma \cdot \partial \psi_{\nu} \\
\mathcal{L}_{N N \gamma}= & N^{\dagger} i v \cdot D N \\
& +\frac{1}{2 M_{N}} N^{\dagger}\left((v \cdot D)^{2}-D^{2}-\frac{i}{2}\left[S^{\alpha}, S^{\beta}\right]\left(\mu_{V} \tilde{f}_{\alpha \beta}^{+}+\frac{1}{2} \mu_{S} \operatorname{Tr}\left[f_{\alpha \beta}^{+}\right]\right)\right) N \\
\mathcal{L}_{N N e \nu}= & -\frac{G_{F} V_{u d}}{\sqrt{2}} \bar{\psi}_{e} \gamma_{\alpha}\left(1-\gamma_{5}\right) \psi_{\nu}\left(N^{\dagger} \tau^{+}\left[v^{\alpha}-2 g_{A} S^{\alpha}\right] N\right. \\
& \left.+\frac{1}{2 M_{N}} N^{\dagger} \tau^{+}\left(i\left(v^{\alpha} v^{\beta}-g^{\alpha \beta}-2 g_{A} v^{\alpha} S^{\beta}\right)(\overleftarrow{\partial}-\vec{\partial})_{\beta}-2 i \mu_{V}\left[S^{\alpha}, S \cdot(\overleftarrow{\partial}+\vec{\partial})\right]\right) N\right)
\end{aligned}
$$

where $F_{\alpha \beta}=\partial_{\alpha} A_{\beta}-\partial_{\beta} A_{\alpha}$ and $D_{\alpha}$ is the covariant derivative of QED. $\xi_{A}$ is the gauge parameter and we choose the Feynman gauge $\xi_{A}=1 . v^{\alpha}$ is the velocity vector with the condition $v^{2}=1$. In the rest frame of the neutron, $v^{\mu}=(1, \overrightarrow{0})$ and $2 S^{\mu}=(0, \vec{\sigma})$. Furthermore, the photon couples to the nucleon via the charge operator $\mathcal{Q}=\frac{1}{2}\left(1+\tau_{3}\right)$, giving $f_{\alpha \beta}^{+}=2 \mathcal{Q} F_{\alpha \beta}$ and $\tilde{f}^{+} \equiv f^{+}-\frac{1}{2} \operatorname{Tr}\left[f^{+}\right] . \mu_{V}$ and $\mu_{S}$ are isovector and isoscalar nucleon magnetic moments; $\mu_{V}=\mu_{p}-\mu_{n}=4.706$.

The counting rules discussed above implicitly assume that higher-order terms in the Lagrangian are suppressed by successively more powers of $\Lambda_{\chi} \approx M_{N}$. In reality however the break-down scale of the EFT is governed by the lightest degree of freedom which has been (at least conceptually) integrated out. Here, this is the pion, which is substantially lighter than $\Lambda_{\chi}$. The low-energy constants (LECs) of the pionless theory may therefore be governed by inverse powers of the pion mass, and hence larger than expected.

To the order we are working, the only LECs which enter are coupling constants such as $g_{A}$ and the magnetic moments, and these are fixed by their well-known experimental values. At higher orders though, potentially $1 / m_{\pi}$-enhanced form-factor terms start to show up. In fact at the next order there is only one such term, the induced pseudoscalar form factor of the nucleon. This is discussed in the final section.

\section{AMPLITUDES}

The relevant Feynman diagrams for the NNLO calculation are shown in Figs. 1, 2, and 3, A contribution to the $D$ coefficient is obtained from the interference between the imaginary part of an amplitude from a loop diagram and the real amplitude of a tree-level diagram [1, 8]. Fig. 1 contains the tree diagrams and those loop diagrams which have an imaginary part and which contribute to the $D$ coefficient; these together give the $\mathcal{O}\left(\alpha \bar{Q} / M_{N}\right)$ contribution. The others do not contribute, in the case of Fig. 2 because they have no imaginary part. The diagrams of Fig. 3 (which with the leading tree graph would give $\mathcal{O}\left(\alpha^{2} / 2 \pi\right)$ contributions) will be considered in section 5 . There is no $\mathcal{O}\left(\left(\bar{Q} / M_{N}\right)^{2}\right)$ contribution since this comes from tree amplitudes only.

We first consider the tree-level amplitudes. The LO amplitude from the diagram (a) in 


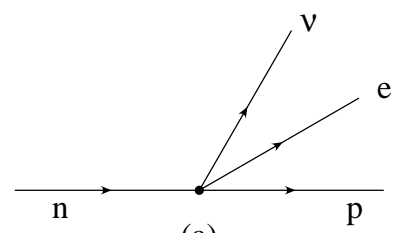

(a)

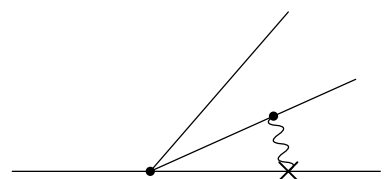

(d)

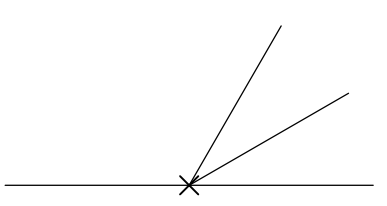

(b)

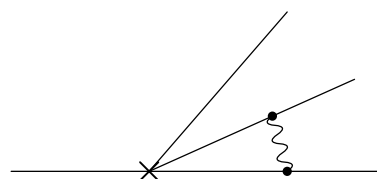

(e)

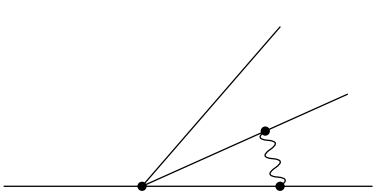

(c)

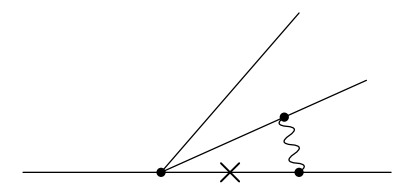

(f)

FIG. 1: Diagrams for the $\mathcal{O}\left(\alpha \bar{Q} / M_{N}\right)$ contributions to the $D$ coefficient. In all diagrams the LO nucleon vertices are denoted with a dot, and NLO ones $\left(\mathcal{O}\left(\bar{Q} / M_{N}\right)\right)$ with a cross. A wavy line denotes a photon. Diagrams (a) and (b) are tree-level LO and NLO diagrams. Diagram (c) is NLO $(\mathcal{O}(\alpha 2 \pi))$ while diagrams (d), (e), and (f) are NNLO as they include $\mathcal{O}\left(\bar{Q} / M_{N}\right)$ corrections.

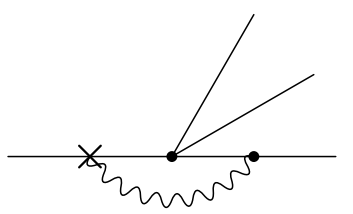

(g)

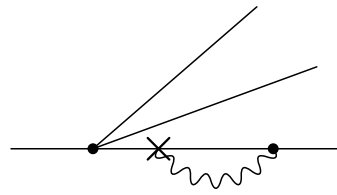

(k)

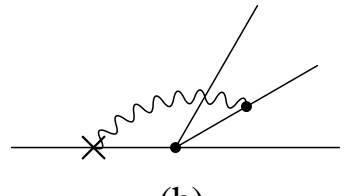

(h)

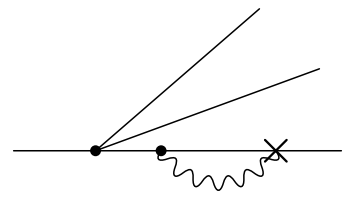

(1)

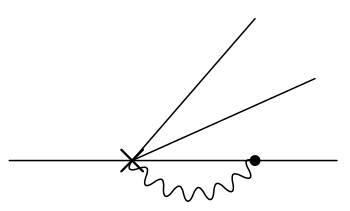

(i)

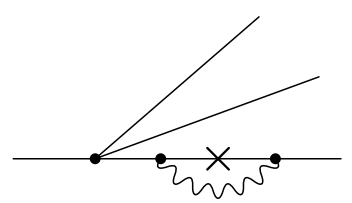

(m)

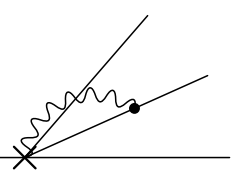

(j)

FIG. 2: Diagrams for the $\mathcal{O}\left(\alpha \bar{Q} / M_{N}\right)$ amplitudes which do not contribute to the $D$ coefficient. See the caption of Fig. 1 for the details.

Fig. 1 reads

$$
\begin{aligned}
M_{(a)} & =\frac{G_{F} V_{u d}}{\sqrt{2}} \bar{u}_{e}\left(p_{e}\right) \gamma_{\alpha}\left(1-\gamma_{5}\right) v_{\nu}\left(p_{\nu}\right) \chi_{p}^{\dagger}\left(v^{\alpha}-2 g_{A} S^{\alpha}\right) \chi_{n} \\
& \equiv \frac{G_{F} V_{u d}}{\sqrt{2}} M_{0}
\end{aligned}
$$

The $\mathcal{O}\left(\bar{Q} / M_{N}\right)$ tree-level amplitude from the diagram (b) in Fig. 1 reads

$$
\begin{aligned}
M_{(b)}= & \frac{G_{F} V_{u d}}{\sqrt{2}} \bar{u}_{e}\left(p_{e}\right) \gamma_{\alpha}\left(1-\gamma_{5}\right) v_{\nu}\left(p_{\nu}\right) \\
& \times \frac{1}{2 M_{N}} \chi_{p}^{\dagger}\left(\left(g^{\alpha \beta}-v^{\alpha} v^{\beta}+2 g_{A} v^{\alpha} S^{\beta}\right)\left(p_{p}+p_{n}\right)_{\beta}+2 \mu_{V}\left[S^{\alpha}, S \cdot\left(p_{p}-p_{n}\right)\right]\right) \chi_{n},
\end{aligned}
$$

where $p_{p}^{\alpha}$ and $p_{n}^{\alpha}$ are the residual four-momenta for proton and neutron, respectively. We define them as $p_{n, p}^{\alpha}=P_{n, p}^{\alpha}-m_{p} v^{\alpha}$ where $P_{n}^{\alpha}$ and $P_{p}^{\alpha}$ are four-momenta for neutron and proton, respectively: $P_{n}^{2}=m_{n}^{2}$ and $P_{p}^{2}=m_{p}^{2}$. 


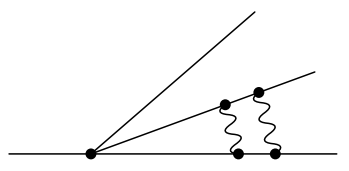

(A)

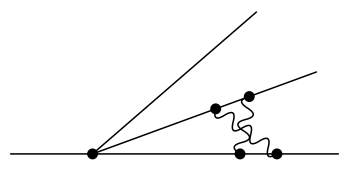

(B)

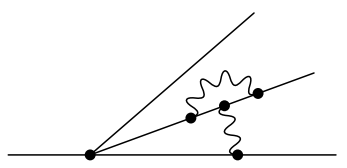

(C)

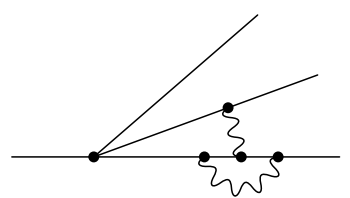

(D)

FIG. 3: The non-trivial diagrams for the $\mathcal{O}\left(\alpha^{2} / 2 \pi\right)$ amplitudes. See the caption of Fig. 1 for details.

We now consider the loop diagrams in Fig. 1. The diagram (c) in Fig. 1 has already been calculated in Ref. [15] giving

$$
M_{(c)}=e^{2} \frac{G_{F} V_{u d}}{\sqrt{2}}\left(M_{0} F_{0}+M_{1}\left(-m_{e} f_{2}\right)\right)
$$

with

$$
\begin{aligned}
M_{1} & =\bar{u}_{e}\left(p_{e}\right) \psi \gamma^{\alpha}\left(1-\gamma_{5}\right) v_{\nu}\left(p_{\nu}\right) \chi_{p}^{\dagger}\left(v_{\alpha}-2 g_{A} S_{\alpha}\right) \chi_{n}, \\
F_{0} & =f_{1}+2 E_{e}\left(f_{2}-f_{0}\right),
\end{aligned}
$$

where $f_{0}, f_{1}$, and $f_{2}$ are loop functions whose definitions are given in Appendix A. From the diagrams (d), (e), and (f) in Fig. 1, we have

$$
\begin{aligned}
M_{(d)}= & -\frac{G_{F} V_{u d}}{\sqrt{2}} \frac{e^{2}}{2 M_{N}} \int_{l} \frac{\bar{u}_{e} \gamma^{\mu}\left(\not p_{e}-\not+m_{e}\right) \gamma^{\alpha}\left(1-\gamma_{5}\right) v_{\nu}}{v \cdot l l^{2}\left(l^{2}-2 p_{e} \cdot l\right)} \\
& \times \chi_{p}^{\dagger}\left(\left(g_{\mu \nu}-v_{\mu} v_{\nu}\right)\left(l+2 p_{p}\right)^{\nu}-2 \mu_{p}\left[S_{\mu}, S \cdot l\right]\right)\left(v_{\alpha}-2 g_{A} S_{\alpha}\right) \chi_{n}, \\
M_{(e)}= & -\frac{G_{F} V_{u d}}{\sqrt{2}} \frac{e^{2}}{2 M_{N}} \int_{l} \frac{\bar{u}_{e} \psi\left(p_{e}-\not+m_{e}\right) \gamma^{\alpha}\left(1-\gamma_{5}\right) v_{\nu}}{v \cdot l l^{2}\left(l^{2}-2 p_{e} \cdot l\right)} \\
& \times \chi_{p}^{\dagger}\left(g_{\alpha \beta}-v_{\alpha} v_{\beta}+2 g_{A} v_{\alpha} S_{\beta}+2 \mu_{V}\left[S_{\alpha}, S_{\beta}\right]\right)\left(l+p_{p}+p_{n}\right)^{\beta} \chi_{n}, \\
M_{(f)}= & -\frac{G_{F} V_{u d}}{\sqrt{2}} \frac{e^{2}}{2 M_{N}} \int \frac{\bar{u}_{e} \psi\left(\not p_{e}-l+m_{e}\right) \gamma^{\alpha}\left(1-\gamma_{5}\right) v_{\nu}}{(v \cdot l)^{2} l^{2}\left(l^{2}-2 p_{e} \cdot l\right)}\left(v \cdot l^{2}-l^{2}-2 p_{p} \cdot l\right) \\
& \times \chi_{p}^{\dagger}\left(v_{\alpha}-2 g_{A} S_{\alpha}\right) \chi_{n},
\end{aligned}
$$

with

$$
\int_{l} \equiv \frac{1}{i} \int \frac{d^{4} l}{(2 \pi)^{4}}
$$

where we have used the fact that the nucleon kinetic energy is $\mathcal{O}\left(\bar{Q}^{2} / M_{N}\right)$ to set $v \cdot p_{p} \simeq 0$ in the nucleon propagator.

\section{THE $D$ COEFFICIENT FROM HEAVY-BARYON EFT}

In calculating the decay rate, which is proportional to the modulus of the amplitude squared, we are taking the product of one diagram and the time-reversed version of another, e.g. 
$M_{(a)} M_{(d)}^{*}$. Considering the same pair in the opposite order, $M_{(d)} M_{(a)}^{*}$, it is obvious that the time-reversal odd term $i \hat{n} \cdot\left(\vec{p}_{e} \times \vec{p}_{\nu}\right)$ will have the opposite sign and a complex-conjugated coefficient compared with the original order. Thus when we sum the two, we can only get a non-zero result if the coefficient has an imaginary part, and this must come from a loop integral with a physical intermediate state.

From the interference between the LO amplitude from the diagram (a) and the imaginary part of the leading loop diagram (c), we have as the potential NLO contribution to the $D$ coefficient

$$
D \propto \sum_{\text {spin }}^{D}\left(M_{(c)} M_{(a)}^{*}+M_{(a)} M_{(c)}^{*}\right)=0,
$$

where the superscript $D$ on the summation denotes that we sum over the spins of the the electron, neutrino, and proton but not that of the neutron, and keep only terms contributing to the $D$ coefficient, those proportional to $\hat{n} \cdot\left(\vec{p}_{e} \times \vec{p}_{\nu}\right)$ where $\hat{n}=\chi_{n}^{\dagger} \vec{\sigma} \chi_{n}$. (The corresponding 4 -vector spin polarisation $N^{\mu}$ is given by $N^{\mu}=\chi_{n}^{\dagger} 2 S^{\mu} \chi_{n}$, and $i \epsilon^{\mu \nu \alpha \beta} v_{\mu} N_{\nu} p_{e \alpha} p_{\nu \beta}=\hat{n} \cdot\left(\vec{p}_{e} \times\right.$ $\left.\vec{p}_{\nu}\right)$.) This NLO term vanishes because there is no such term in $\sum_{\text {spin }} M_{0}^{*} M_{0,1}$, and not through details of the loop integrals $F_{0}$ and $f_{1}$. Thus the $D$ coefficient has no contribution up to NLO.

We now consider $\mathcal{O}\left(\alpha \bar{Q} / M_{N}\right)$ interference terms. From the amplitudes from the NLO $\left(\mathcal{O}\left(\bar{Q} / M_{N}\right)\right)$ diagram (b) and leading loop diagram (c), we have

$$
\begin{aligned}
\sum_{\text {spin }}^{D}\left(M_{(c)} M_{(b)}^{*}\right. & \left.+M_{(b)} M_{(c)}^{*}\right)=-4\left(G_{F} V_{u d}\right)^{2} \frac{e^{2}}{2 M_{N}} \epsilon^{\mu \nu \alpha \beta} v_{\mu} N_{\nu} p_{e \alpha} p_{\nu \beta} \\
\times & \left\{2 \operatorname{Im} F_{0}\left(E_{e}\left(-g_{A} \mu_{V}+g_{A}+g_{A}^{2}-\mu_{V}\right)+E_{\nu}\left(-g_{A} \mu_{V}+g_{A}-g_{A}^{2}+\mu_{V}\right)\right)\right. \\
& \left.+2 \operatorname{Im} f_{2} m_{e}^{2}\left(g_{A} \mu_{V}-g_{A}-g_{A}^{2}+\mu_{V}\right)\right\} .
\end{aligned}
$$

From the LO amplitude $M_{(a)}$ and the NNLO $\left(\mathcal{O}\left(\alpha \bar{Q} / M_{N}\right)\right.$ amplitudes $M_{(d, e, f)}$, we have

$$
\begin{aligned}
& \sum_{\text {spin }}^{D}\left(M_{(d)} M_{(a)}^{*}+M_{(a)} M_{(d)}^{*}\right)=-4\left(G_{F} V_{u d}\right)^{2} \frac{e^{2}}{2 M_{N}} \epsilon^{\mu \nu \alpha \beta} v_{\mu} N_{\nu} p_{e \alpha} p_{\nu \beta} \\
& \times\left(1-g_{A}\right)\left(-8 g_{A} E_{\nu} \operatorname{Im} f_{1}+2 \mu_{p}\left(1+3 g_{A}\right)\left(2 \operatorname{Im} f_{4}+E_{e} \operatorname{Im} f_{5}+m_{e}^{2} \operatorname{Im} f_{6}\right)\right), \\
& \sum_{\text {spin }}^{D}\left(M_{(e)} M_{(a)}^{*}+M_{(a)} M_{(e)}^{*}\right)=-4\left(G_{F} V_{u d}\right)^{2} \frac{e^{2}}{2 M_{N}} \epsilon^{\mu \nu \alpha \beta} v_{\mu} N_{\nu} p_{e \alpha} p_{\nu \beta} \\
& \left\{\times 2 \operatorname{Im} F_{0}\left(E_{e}\left(g_{A} \mu_{V}-g_{A}-g_{A}^{2}+\mu_{V}\right)+E_{\nu}\left(g_{A} \mu_{V}-g_{A}+g_{A}^{2}-\mu_{V}\right)\right)\right. \\
& +2\left(g_{A} \mu_{V}-g_{A}-g_{A}^{2}+\mu_{V}\right)\left(-E_{e} \operatorname{Im} f_{5}+\left(-m_{e}^{2}+2 E_{e}^{2}\right)\left(\operatorname{Im} f_{2}-\operatorname{Im} f_{6}\right)\right) \\
& \left.+4\left(\mu_{V}-g_{A}\right) \operatorname{Im} f_{4}\right\} \text {, } \\
& \sum_{\text {spin }}^{D}\left(M_{(f)} M_{(a)}^{*}+M_{(a)} M_{(f)}^{*}\right)=-4\left(G_{F} V_{u d}\right)^{2} \frac{e^{2}}{2 M_{N}} \epsilon^{\mu \nu \alpha \beta} v_{\mu} N_{\nu} p_{e \alpha} p_{\nu \beta}\left(-8 g_{A}\left(1-g_{A}\right) E_{\nu} \operatorname{Im} g_{4}\right) \text {, }
\end{aligned}
$$

where explicit expressions for the loop functions $f_{i}$ and $g_{4}$ are given in the Appendix. (Throughout, we have used unit normalization for the heavy-baryon nucleon spinors). 
To obtain the $D$ coefficient, we have to divide out the common factor $4\left(G_{F} V_{u d}\right)^{2} E_{e} E_{\nu}\left(1+3 g_{A}^{2}\right)$ which also appears in the angle-independent term in the leading matrix element squared, $\sum_{\text {spin }} M_{(a)}^{*} M_{(a)}$.

We thus have the final expression for the $\mathcal{O}\left(\alpha \bar{Q} / M_{N}\right)$ contribution to the $D$ coefficient for neutron beta decay:

$$
\begin{aligned}
D_{\mathrm{CT}}=\frac{1}{1+3 g_{A}^{2}} \frac{\alpha E_{e}}{4 M_{N}} & \frac{1}{\beta}\left(\left(1+3 g_{A}\right)\left(\left(\mu_{V}-g_{A}\right)-3 \mu_{p}\left(1-g_{A}\right)\right)\right. \\
& \left.+\frac{m_{e}^{2}}{E_{e}^{2}}\left(\left(3+g_{A}\right)\left(\mu_{V}-g_{A}\right)+3 \mu_{p}\left(1-g_{A}\right)\left(1+3 g_{A}\right)\right)\right) .
\end{aligned}
$$

where $\beta \equiv\left|\vec{p}_{e}\right| / E_{e}$. This is exactly the result obtained by Callan and Treiman [8].

\section{TERMS HIGHER-ORDER IN $\alpha$}

Since, as discussed above, the two scales $\alpha / 2 \pi$ and $\bar{Q} / M_{N}$ are of the same size, $\mathcal{O}\left(\alpha^{2} / 2 \pi\right)$ terms, if they exist, could be as large as the well-known $\mathcal{O}\left(\alpha \bar{Q} / M_{N}\right)$ contribution which we reproduced in the previous section. We therefore need to check these too. The relevant diagrams are shown in Fig. 3, and their amplitudes are as follows:

$$
\begin{aligned}
M_{(A)} \propto & \int_{l} \int_{k} \frac{\bar{u}_{e}\left(p_{e}\right) \psi\left(\not p_{e}+\not k+m_{e}\right) \psi\left(\not p_{e}+\not+m_{e}\right) \gamma_{\alpha}\left(1-\gamma_{5}\right) v_{\nu}\left(p_{\nu}\right)}{v \cdot l v \cdot k k^{2}(k-l)^{2}\left(l^{2}-2 p_{e} \cdot l\right)\left(k^{2}-2 p_{e} \cdot k\right)} \chi_{p}^{\dagger}\left(v^{\alpha}-2 g_{A} S^{\alpha}\right) \chi_{n}, \\
M_{(B)} \propto & \int_{l} \int_{k} \frac{\bar{u}_{e}\left(p_{e}\right) \psi\left(\not p_{e}+\not k+m_{e}\right) \psi\left(\not p_{e}+l+m_{e}\right) \gamma_{\alpha}\left(1-\gamma_{5}\right) v_{\nu}\left(p_{\nu}\right)}{v \cdot l v \cdot(l-k) k^{2}(k-l)^{2}\left(l^{2}-2 p_{e} \cdot l\right)\left(k^{2}-2 p_{e} \cdot k\right)} \chi_{p}^{\dagger}\left(v^{\alpha}-2 g_{A} S^{\alpha}\right) \chi_{n}, \\
M_{(C)} \propto & \int_{l} \int_{k} \frac{\bar{u}_{e}\left(p_{e}\right) \gamma^{\sigma}\left(\not p_{e}+\not k+m_{e}\right) \psi\left(p_{e}+\not+\not k+m_{e}\right) \gamma_{\sigma}\left(\not p_{e}+\not+m_{e}\right) \gamma_{\alpha}\left(1-\gamma_{5}\right) v_{\nu}\left(p_{\nu}\right)}{v \cdot l l^{2} k^{2}\left(l^{2}-2 p_{e} \cdot l\right)\left(k^{2}-2 p_{e} \cdot k\right)\left((l+k)^{2}-2 p_{e} \cdot(l+k)\right)} \\
& \times \chi_{p}^{\dagger}\left(v^{\alpha}-2 g_{A} S^{\alpha}\right) \chi_{n}, \\
M_{(D)} \propto & \int_{l} \int_{k} \frac{\bar{u}_{e}\left(p_{e}\right) \psi\left(\not p_{e}+\not l+m_{e}\right) \gamma_{\alpha}\left(1-\gamma_{5}\right) v_{\nu}\left(p_{\nu}\right)}{v \cdot l v \cdot(l+k) v \cdot k l^{2} k^{2}\left(l^{2}-2 p_{e} \cdot l\right)} \chi_{p}^{\dagger}\left(v^{\alpha}-2 g_{A} S^{\alpha}\right) \chi_{n} .
\end{aligned}
$$

Clearly the structure of the amplitude $M_{(D)}$ is the same as the leading one-photon-loop diagram $M_{(c)}$, though the integral is different. So interference with $M_{0}$ cannot give a contribution to the $D$ coefficient. The other three cases are more complicated (though it is worth noticing that the tensor structures of $M_{(A)}$ and $M_{(B)}$ are the same; again only the integrals differ). However explicit calculation - without the need actually to calculate any scalar two-loop integrals - again shows a vanishing contribution to the $D$ coefficient. (The calculations are most efficiently done using e.g. the package "Tracer" on Mathematica [20]; we do not give details of the reduction to scalar integrals as in the Appendix because they are not required.)

Having shown through explicit calculation that there are no two-photon-loop contributions to the $D$ coefficient in the heavy-baryon limit, it is interesting to consider if this can be generalized. The crucial features which make it possible are that in this limit, as can be seen in the expressions for the diagrams (c) and (A-D), the amplitude factorizes into a hadronic and leptonic part. Since the leading-order photon coupling to the proton is spin-independent, no matter how complicated the photon exchanges and dressings are, the hadronic tensor is unchanged. Furthermore, since the heavy-baryon propagator does not 
depend on the three-momentum of the proton, the loop integrals can only pick up factors of the electron momentum and the velocity vector, limiting the complexity of the structures which can appear in the leptonic tensor. Thus it is plausible that many-photon effects do not in fact generate new structures. Recall that the vanishing of the $D$ coefficient in the oneand two-photon-loop case is due to the tensor structure, and not to details of the integrals involved.

In fact it was shown long ago that one class of corrections vanish to all order in $\alpha$, namely the repeated exchange of Coulomb photons [16]. In the heavy-baryon limit these only introduce a phase shift in the final-state wavefunction, and cannot induce a $D$ coefficient. However in the relativistic theory crossed photons, or multiple overlapping dressings of either fermion line, cannot be accounted for so easily as the interaction kernel becomes arbitrarily complicated. In the heavy-baryon formalism however the complexity is much reduced, as indicated above. As we will demonstrate in what follows, we can show that no corrections survive at any order.

The amplitude resulting from the exchange of any number of (non-magnetic) photons (crossed or not) between the electron and proton, and from photon-loop dressing of one or more photon-electron or photon-proton vertices, has the same general form as the single photon diagram (c), albeit with multiple integrals and many insertions along the electron line. The basic structure can be written schematically as follows:

$$
M_{X} \propto\left(\int_{l_{1}} \ldots \int_{l_{n}} \frac{\bar{u}_{e}\left(p_{e}\right) \hat{O}\left(\left\{l_{i}\right\}\right) \gamma_{\alpha}\left(1-\gamma_{5}\right) v_{\nu}\left(p_{\nu}\right)}{v \cdot l_{i}^{\prime} \ldots l_{j}^{\prime 2} \ldots\left(l_{k}^{\prime 2}-2 p_{e} \cdot l_{k}^{\prime}\right) \ldots}\right) \chi_{p}^{\dagger}\left(v^{\alpha}-2 g_{A} S^{\alpha}\right) \chi_{n},
$$

where the $l_{i}^{\prime}$ are linear combinations of the loop momenta $l_{i}$, and $\hat{O}\left(\left\{l_{i}\right\}\right)$ is composed only of the building blocks $m_{e},\left\{l_{i}\right\}, p_{e}, \psi$ and $\gamma_{\sigma} \ldots \gamma^{\sigma}$ (the last being for a photon loop dressing one or more vertices, and with ... standing for more of the same).

When the loop integrals are performed, all loop momenta $l_{i}^{\alpha}$ in the numerator either become $p_{e}^{\alpha}$ or $v^{\alpha}$, or a pair gives $l_{i}^{\alpha} l_{j}^{\beta} \rightarrow g^{\alpha \beta}$. Thus after integration, the whole structure represented by $\hat{O}\left(\left\{l_{i}\right\}\right)$ can only give a small number of terms, namely $I, p_{e}, \psi$ and $\left[p_{e}, \psi\right]$, and the same multiplied by $\gamma_{5}$. Furthermore since $\gamma_{5} \gamma_{\alpha}\left(1-\gamma_{5}\right)=\gamma_{\alpha}\left(1-\gamma_{5}\right)$, the structures with $\gamma_{5}$ in them are redundant. Hence the bottom line is

$$
M_{X}=\sum_{n=1}^{4} I_{n}^{X}\left(m_{e}, v \cdot p_{e}\right) \bar{u}_{e}\left(p_{e}\right) \hat{O}_{n} \gamma_{\alpha}\left(1-\gamma_{5}\right) v_{\nu}\left(p_{\nu}\right) \chi_{p}^{\dagger}\left[v^{\alpha}-2 g_{A} S^{\alpha}\right] \chi_{n},
$$

where the integrals $I_{n}^{X}$ will depend on the particular graph we are considering, and the operators $\hat{O}_{n}$ are the four listed above.

When we calculate the decay rate from the amplitude we need expressions like $M_{X}^{\dagger} M_{Y}$, with a sum over the spin of the proton, electron and neutrino. Using completeness relations such as $\sum_{s} u_{e}\left(p_{e}, s\right) \bar{u}_{e}\left(p_{e}, s\right)=p_{e}+m_{e}$, we end up with terms like the following:

$$
\begin{gathered}
M_{X}^{\dagger} M_{Y}=\sum_{m, n=1}^{4}\left(I_{m}^{X}\right)^{*} I_{n}^{Y} \operatorname{Tr}\left[\gamma_{\alpha}\left(1-\gamma_{5}\right) \bar{O}_{m}\left(p_{e}+m_{e}\right) \hat{O}_{n} \gamma_{\beta}\left(1-\gamma_{5}\right) p_{\nu}\right] \times \\
\chi_{n}^{\dagger}\left(v^{\alpha}-2 g_{A} S^{\alpha}\right)\left(v^{\beta}-2 g_{A} S^{\beta}\right) \chi_{n},
\end{gathered}
$$

where $\bar{O}_{m}=\gamma_{0} \hat{O}_{m}^{\dagger} \gamma_{0}= \pm \hat{O}_{m}$. Finally, we note that $\bar{O}_{m}\left(\not_{e}+m_{e}\right) \hat{O}_{n}$ is itself just a combination of the $\hat{O}_{m}$, giving just four structures to be calculated:

$$
\operatorname{Tr}\left[\gamma_{\alpha}\left(1-\gamma_{5}\right) \hat{O}_{n} \gamma_{\beta}\left(1-\gamma_{5}\right) p_{\nu}\right] \chi_{n}^{\dagger}\left(v^{\alpha}-2 g_{A} S^{\alpha}\right)\left(v^{\beta}-2 g_{A} S^{\beta}\right) \chi_{n} .
$$


Only $\hat{O}_{n}=\not p_{e}$ and $\psi$ give non-vanishing results, and they do not generate the structure $\epsilon_{\alpha \beta \sigma \tau} p_{e}^{\alpha} p_{\nu}^{\beta} v^{\sigma} \chi_{n}^{\dagger} S^{\tau} \chi_{n}$. So in the heavy-baryon limit, there are no contributions to the $D$ coefficient at any order in $\alpha$. Corrections to the leading $\alpha \bar{Q} / M_{N}$ result will be $\mathcal{O}\left(\alpha^{2} \bar{Q} / 2 \pi M_{N}\right)$ and $\mathcal{O}\left(\alpha \bar{Q}^{2} / M_{N}^{2}\right)$ - presumably at least another factor of $10^{-3}$ down. ${ }^{3}$

\section{HIGHER-ORDER CORRECTIONS AND CONCLUSIONS}

In the preceding sections, we have shown that the Callan-Treiman result for the $D$ coefficient in neutron $\beta$ decay is the leading non-vanishing contribution in a heavy-baryon EFT in which the expansion parameters, $\alpha / 2 \pi$ and $\bar{Q} / M_{N}$, are both of the order of $10^{-3}$. The non-zero contribution to the $D$ coefficient appears because of the electromagnetic finalstate interaction at $\mathcal{O}\left(\alpha \bar{Q} / M_{N}\right)$, whereas there are no contributions from the $\mathcal{O}\left(\alpha^{2} / 2 \pi\right)$ and $\mathcal{O}\left(\left(\bar{Q} / M_{N}\right)^{2}\right)$ terms. The $D$ coefficient is NNLO with respect to the decay rate, and hence very small $\left(\sim 10^{-5}\right)$. Nonetheless there are hopes that this might be experimentally accessible in the not-too-distant future. The interest in such experiments would, of course, be to detect deviations which might indicate new physics, and to interpret such a result the accuracy of the Callan-Treiman prediction must be known. At first glance our results suggest that the next correction would introduce a relative error of order $10^{-3}$, which should be small enough to be unimportant for many years to come; in particular this would be small enough to allow detection (if sufficiently sensitive experiments could be carried out) of the prediction from the MSSM of a contribution to the $D$ coefficient of the order of $10^{-7}[3]$.

However at the next order $\left(\mathrm{N}^{3} \mathrm{LO}\right)$ there will be contributions from the third-order Lagrangian, and these will not only be the $1 / M_{N}^{2}$ terms required by Lorentz invariance, but will also include new structures whose scale is governed by the lightest degrees of freedom which have been integrated out - in this case the pion. Potentially therefore the corrections to the $\mathcal{O}\left(\alpha \bar{Q} / M_{N}\right)$ result are $\mathcal{O}\left(\alpha \bar{Q}^{2} / m_{\pi}^{2}\right)$, giving a relative error of perhaps $5 \%$.

Looking at the diagrams of Fig. 1, such insertions from the third-order Lagrangian could in principle replace any of the crosses, though that in Fig. If could only give a proton mass shift. However because we need to take the imaginary part of the loop graphs, the nucleon is on-shell everywhere and most of the vertex corrections are just form-factor corrections to the structures we have already considered-e.g. $\mu_{p} \rightarrow \mu_{p}\left(1+\left\langle r_{M}^{2}\right\rangle q^{2} / 6\right)$-which are therefore two orders down. ${ }^{4}$ Only one potentially-enhanced third-order effect remains, and that is where the cross in Fig. 1b and Fig. 1e represents the pseudoscalar form factor of the nucleon, the leading contribution to which is given by the exchange of a pion between the nucleon and leptons.

This pseudoscalar vertex is easily calculated from HB $\chi \mathrm{PT}$ to be as follows (where $\left(q^{2}-m_{\pi}^{2}\right)$ in the pion propagator has been replaced with $-m_{\pi}^{2}$ since the momentum transfer is very

3 The referee of this paper has drawn our attention to the paper by Gross [21] on two particles of unequal mass interacting via relativistic exchange of some boson, and in particular to the effective one-body Dirac equation obeyed by the light particle when the heavy particle's mass is taken to infinity. It is possible that the all-orders vanishing of electromagnetic contributions discussed here may also be explicable within that framework.

${ }^{4}$ If we work in $\mathrm{HB} \chi \mathrm{PT}$ with explicit pions, these form factors arise partly from pion loops. The leading term which is $\mathcal{O}\left(\bar{Q} m_{\pi} /\left(4 \pi f_{\pi}^{2}\right)\right.$ simply renormalises the isovector magnetic moment and the next term in an expansion in the photon momentum transfer gives a term of relative size $\bar{Q}^{2} / m_{\pi}^{2}$. 
much less than $\left.m_{\pi}\right)$ :

$$
-i M_{\mathrm{PS}}=i \frac{G_{F} V_{u d}}{\sqrt{2}} \frac{2 g_{A}}{m_{\pi}^{2}} q_{\alpha} S \cdot q \gamma^{\alpha}\left(1-\gamma_{5}\right),
$$

There are two contributions to the $D$ coefficient, arising from the interference of the amplitudes (1a) and (1e), and of (1b) and (1c) (with the cross in (1b) and (1e) now representing the new vertex). These give a final result of

$$
\begin{aligned}
D_{\pi} & =-\frac{1}{1+3 g_{A}^{2}} \frac{e^{2} m_{e}^{2}}{m_{\pi}^{2}} g_{A}^{2}\left(-4\left(E_{e}+E_{\nu}\right) \operatorname{Im} f_{2}+2 \operatorname{Im} f_{5}+2 E_{e} \operatorname{Im} f_{6}\right) \\
& =-\frac{1}{1+3 g_{A}^{2}} \frac{\alpha m_{e}^{2}}{m_{\pi}^{2}} \frac{g_{A}^{2}}{\beta} \frac{2\left(E_{e}+E_{\nu}\right)}{E_{e}} .
\end{aligned}
$$

With the approximation $E_{e}+E_{\nu}=m_{n}-m_{p}$ this has the value $-5.88\left(p_{e}^{\max } / p_{e}\right) \times 10^{-8}$ compared to the leading result of $\left(0.228\left(p_{e}^{\max } / p_{e}\right)+1.083\left(p_{e} / p_{e}^{\max }\right)\right) \times 10^{-5}$ - a correction of between $-0.5 \%$ and $-2.5 \% .^{5}$

With the only $\left(1 / m_{\pi}\right)$-enhanced $\mathrm{N}^{3} \mathrm{LO}$ term explicitly calculated, we can now say with confidence that our final result for the $D$ coefficient,

$$
D=D_{\mathrm{CT}}+D_{\pi}
$$

where $D_{\mathrm{CT}}$ and $D_{\pi}$ are given by Eqs (20) and (27), should be accurate to better than $1 \%$, with expected corrections being $\mathcal{O}\left(10^{-3}\right)$.

\section{Acknowledgments}

SA would like to thank J Behr and H M Shimizu for discussions, H-W Fearing and M Igarashi for communications. JAMcG would like to thank M Birse for discussions and for reading the manuscript and $\mathrm{T}$ Cohen for discussions. The work of SA and JAMcG is supported by STFC grant number PP/F000448/1. The work of TS is supported by the Japan Society for the Promotion of Science, Grant-in-Aid for Scientific Research(c) 20540270.

\section{Appendix: Loop functions}

The loop functions for the $D$-calculation in $\mathrm{HB} \chi \mathrm{PT}$ are defined as

$$
\int_{l} \frac{1}{(v \cdot l+i \eta)\left(l^{2}+i \eta\right)\left(l^{2}-2 p_{e} \cdot l+i \eta\right)}=f_{0}
$$

where

$$
\int_{l} \equiv \frac{\mu^{4-d}}{i} \int \frac{d^{d} l}{(2 \pi)^{d}}
$$

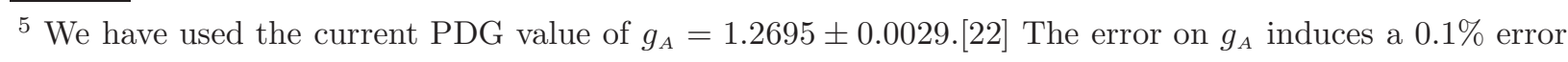
in $D$ at $p_{e}=p_{e}^{\max }$. 
and $D$-is the space-time dimensions, $d=4-2 \epsilon$. Furthermore,

$$
\begin{aligned}
\int_{l} \frac{l^{\mu}}{v \cdot l l^{2}\left(l^{2}-2 p_{e} \cdot l\right)} & =v^{\mu} f_{1}+p_{e}^{\mu} f_{2}, \\
\int_{l} \frac{l^{\mu} l^{\nu}}{v \cdot l l^{2}\left(l^{2}-2 p_{e} \cdot l\right)} & =v^{\mu} v^{\nu} f_{3}+g^{\mu \nu} f_{4}+\left(v^{\mu} p_{e}^{\nu}+p_{e}^{\mu} v^{\nu}\right) f_{5}+p_{e}^{\mu} p_{e}^{\nu} f_{6}, \\
\int_{l} \frac{l^{\mu} l^{\nu}}{(v \cdot l)^{2} l^{2}\left(l^{2}-2 p_{e} \cdot l\right)} & =v^{\mu} v^{\nu} g_{3}+g^{\mu \nu} g_{4}+\left(v^{\mu} p_{e}^{\nu}+p_{e}^{\mu} v^{\nu}\right) g_{5}+p_{e}^{\mu} p_{e}^{\nu} g_{6} .
\end{aligned}
$$

where we have suppressed the in terms in the propagators in those expressions. To calculate the contribution to the $D$-term, we need imaginary part of the six loop functions in the followings, and thus have

$$
\begin{aligned}
& \operatorname{Im} f_{1}=-\operatorname{Im} g_{4}=\frac{1}{8 \pi} \frac{1}{\beta}, \quad \operatorname{Im} f_{2}=-\frac{1}{8 \pi} \frac{1}{\beta E_{e}}, \\
& \operatorname{Im} f_{4}=\frac{1}{16 \pi} \beta E_{e}, \quad \operatorname{Im} f_{5}=\frac{1}{16 \pi} \frac{1}{\beta}, \quad \operatorname{Im} f_{6}=-\frac{1}{16 \pi} \frac{1}{\beta E_{e}} .
\end{aligned}
$$

[1] J. D. Jackson, S. R. Treiman, and H. W. Wyld, Jr., Phys. Rev. 106 (1957) 517; Nucl. Phys. 4 (1957) 206.

[2] P. Herczeg and I. B. Khriplovich, Phys. Rev. D 56 (1997) 80.

[3] M. Drees and M. Rauch, Eur. Phys. J. C 29 (2003) 573.

[4] P. Herczeg, Prog. Part. Nucl. Phys. 46 (2001) 413

[5] L. J. Lising et al., Phys. Rev. C 62 (2000) 055501.

[6] T. Soldner et al., Phys. Lett. B 581 (2004) 49.

[7] J. S. Nico and W. M. Snow, Annu. Rev. Nucl. Part. Sci. 55 (2005) 27.

[8] C. G. Callan, Jr. and S. B. Treiman, Phys. Rev. 162 (1967) 1494.

[9] S. Treiman, Annu. Rev. Nucl. Part. Sci. 46 (1996) 1.

[10] J. Erler and M. J. Ramsey-Musolf, Prog. Part. Nucl. Phys. 54 (2005) 351.

[11] S. Weinberg, Physica A 96 (1979) 327.

[12] E. Jenkins and A. V. Manohar, Phys. Lett. B 259 (1991) 353.

[13] G. Müller and U.-G. Meißner, Nucl. Phys. B 556 (1999) 265.

[14] V. Bernard, Prog. Part. Nucl. Phys. 60 (2008) 82.

[15] S. Ando, H. W. Fearing, V. Gudkov, K. Kubodera, F. Myhrer, S. Nakamura, T. Sato, Phys. Lett. B 595 (2004) 250.

[16] J. Brodine Phys. Rev. D 1 (1970) 100.

[17] B. Holstein Phys. Rev. C 5 (1972) 1529.

[18] M. Igarashi, Prog. Theo. Phys. 60 (1978) 1495.

[19] V. Bernard, N. Kaiser, U.-G. Meißner, Phys. Rev. D 50 (1994) 6899.

[20] M. Jamin and M. E. Lautenbacher, Computer Physics Communications 74 (1993) 265.

[21] F. Gross, Phys. Rev. C 26 (1982) 2203.

[22] C. Amsler et al., Phys. Lett. B 667, (2008) 1 